\title{
FOCUS: NEXT GENERATION SEQUENCING
}

\section{Personalized Medicine and Ethics}

\section{DEBORAH JOSKO}

\section{LEARNING OBJECTIVES}

1. Define personalized medicine.

2. Discuss various case scenarios where targeted therapy was initiated based on sequencing results.

3. Describe the advantages and disadvantages of genetic testing.

4. Summarize suggested guidelines when reporting sequencing results.

ABBREVIATIONS: AHQR - Agency for Health Care Research and Quality; ALL - acute lymphoblastic leukemia; AML - acute myeloid leukemia; APL- acute promyelocytic leukemia; ATRA - all-trans retinoic acid; BRAC - breast cancer gene; CANCP - solid tumor targeted cancer gene panel; CLIA - Clinical Laboratory Improvement Amendments; DNA - deoxyribonucleic acid; ERISA - Employee Retirement Income Security Act; FISH - fluorescent in situ hybridization; FDA Food and Drug Administration; FLT3- fetal liver tyrosine kinase 3; HER2 - human epidermal growth factor receptor 2; GINA - Genetic Information Nondiscrimination Act; HIPAA - Health Insurance Portability and Accountability Act; HUGO - human genome organization; IRC - Internal Revenue Code; JAMA - Journal of the American Medical Association; MLO - Medical Laboratory Observer; NGS - next generation sequencing, PGM - Personal Genome Machine, PHSA - Public Health Service Act; RNA ribonucleic acid; WGS - whole genome sequencing

INDEX TERMS: Personalized Medicine, Next Generation Sequencing, Ethics, Sequencing.

\section{Clin Lab Sci 2014;27(3):185}

Deborab Josko, Ph.D. MLT(ASCP)M, SM, Associate Professor and Program Director, Department of Clinical Laboratory Sciences, Medical Laboratory Science Program, Rutgers, The State University of New Jersey - School of Health Related Professions, Scotch Plains, NJ.

Address for correspondence: Deborab Josko, Ph.D.
MLT(ASCP)M, SM, Associate Professor, Department of Clinical Laboratory Sciences, Medical Laboratory Science Program, Rutgers, The State University of New Jersey School of Health Related Professions 1776 Raritan Road, Scotch Plains, NJ 07076. (908) 889-2422. daj100@shrp.rutgers.edu.

\section{Introduction}

The completion of the Human Genome Project along with advances in DNA sequencing techniques and genetic testing have enabled clinicians to diagnose, treat, and monitor a patient's progression through the course of an illness or disease on a personal level. Clinicians now have the ability to counsel individuals and offer preventative options if a genetic test comes back positive. For example, if a woman tests positive for the breast cancer gene (BRAC 1 or BRAC 2) mutation leaving her at high risk for developing breast and/or ovarian cancer, she may decide to undergo a double mastectomy and/or a complete hysterectomy. By having knowledge of one's own genetic makeup, an individual has the ability to take preventative measures in order to lower the risk of developing the illness or disease in the future.

Genetic testing is useful when performed on amniotic fluid to determine if the developing fetus has a particular mutation. If an expecting couple receives news that their baby has the mutation that results in either Down syndrome or Tay Sach's disease, they may decide to abort. However, this scenario does not go without moral and ethical consequences.

This article will provide several case scenarios where next generation sequencing (NGS) was performed and used in treating a patient based on their own genetic make-up or the genetic make-up of the individual's cancer. In addition some of the ethical issues researchers face when using this technology will be discussed.

\section{Definitions of Personalized Medicine}

According to the National Human Genome Research 
Institute, personalized medicine can be defined as, "an emerging practice of medicine that uses an individual's genetic profile to guide decisions made in regard to the prevention, diagnosis, and treatment of disease. Knowledge of a patient's genetic profile can help doctors select the proper medication or therapy and administer it using the proper dose or regimen. Personalized medicine is being advanced through data from the Human Genome Project."

Personalize medicine is possible due to advances in NGS platforms and the applications available for clinical and research use. The various platforms and applications were discussed in the following articles, "Next Generation Sequencing: Platforms" and "Next Generation Sequencing: Applications" that are part of this series.

One application discussed was using NGS to identify various mutations found in oncogenes and tumor suppressor genes which can result in the development of various cancers. The Ion Torrent PGM $^{\mathrm{TM}}$ by Life Technologies offers several cancer panels such as the "Ion AmpliSeq ${ }^{\mathrm{TM}}$ Cancer Hotspot Panel v2 which targets "hot spot" regions of 50 oncogenes and tumor suppressor genes and the Ion AmpliSeq ${ }^{\mathrm{TM}}$ Comprehensive Cancer Panel which targets greater than 400 oncogenes and tumor suppressor genes" ${ }^{2}$ Custom panels are also available as long as the human genome organization (HUGO) symbol or the gene regions based on human genome coordinates are known. ${ }^{3}$ NGS technology will not only revolutionize the way clinicians diagnose and treat patients, particularly oncology patients, but will have a great impact on drug discovery and clinical trials in the future. ${ }^{4}$

The use of NGS in cancer therapy made its debut in 2012 due to advances in this technology. ${ }^{4}$ According to the National Cancer Institute at the National Institutes of Health the definition of personalized medicine as it relates to cancer is, "a form of medicine that uses information about a person's genes, proteins, and environment to prevent, diagnose, and treat disease. In cancer, personalized medicine uses specific information about a person's tumor to help diagnose, plan treatment, find out how well treatment is working, or make a prognosis. Examples of personalized medicine include using targeted therapies to treat specific types of cancer cells, such as human epidermal growth factor receptor 2 (HER2)-positive breast cancer cells, or using tumor marker testing to help diagnose cancer."

\section{Whole Genome Sequencing in Personalized Medicine}

Whole genome sequencing (WGS) involves sequencing the entire human genome in order to identify the type of genetic variations an individual has. Variations such as single nucleotide polymorphisms, inversions, base insertions and deletions, etc. can be detected using this technology. ${ }^{6}$ The main advantage of WGS is that by sequencing the complete genome, mutations in the promoter and regulatory regions can be detected. ${ }^{7}$ This also includes identification of rare mutations which may be missed using target specific therapy. ${ }^{7}$

Conversely, disadvantages have been reported when using WGS on clinical samples. In a study conducted by Dewey et al. at Stanford University Medical Center, the authors demonstrated and concluded from their findings that the use of WGS was "associated with incomplete coverage of inherited disease genes, low reproducibility of detection of genetic variation with the highest potential clinical effects, and uncertainty about clinically reportable findings." ${ }^{8}$ What was interesting to note was the authors did find a frame shift deletion in the BRAC 1 gene in one of the 12 participants in the study as a result of the WGS. Since this mutation put the participant at risk for developing breast and/or ovarian cancer she elected to have preventative surgery and undergo regular cancer screenings to avoid complications in the future. ${ }^{8,9}$

In a study conducted by Welch et al. published in JAMA in 2011, WGS was used which identified a cryptic fusion oncogene not detected using fluorescent in situ hybridization (FISH) or routine cytogenetics. ${ }^{10}$ To summarize this study, a 39-year-old woman in her first remission with acute myeloid leukemia (AML) was referred to the researcher's institution for an allogeneic stem cell transplant, the treatment prescribed when a patient with AML is in their first remission. ${ }^{11}$ Upon further examination, the bone marrow biopsy showed no evidence of AML but cellular morphology was consistent with acute promyelocytic leukemia (APL). After performing WGS on the original bone marrow biopsy, several mutations including deletions, insertions and translocations were observed that were consistent with APL. ${ }^{10}$ WGS techniques enabled scientists to 
identify a cryptic oncogene that was not identified using routine FISH and cytogenetic techniques. ${ }^{10}$ These findings resulted in a change in therapy for the patient which consisted of all-trans retinoic acid (ATRA) used to treat $\mathrm{APL}^{12}$ instead of an allogeneic stem cell transplant performed in patients with AML. ${ }^{11}$ Patient status at the time of the publication indicated the patient was in remission for 15 months after her clinical presentation. ${ }^{10}$

The entire process from the time the DNA samples were collected until the results were reported out to the physician took seven weeks. The authors conclude by stating, "whole-genome sequencing can identify cytogenetically invisible oncogenes in a clinically relevant time frame." ${ }^{10}$ For an in depth account of this case study including the cytogenetics and WGS results, refer to the article entitled, "Use of whole genome sequencing to diagnose a cryptic fusion oncogene" by Welch et al. ${ }^{10}$

\section{Targeted Genotyping Assays}

In a recent publication by Kris et al., scientists found that by identifying oncogenic drivers "(genomic alterations critical to cancer development and maintenance)" they were able to use targeted therapy in patients suffering from lung adenocarcinomas. ${ }^{13}$ They performed multiplexed genotyping assays on tumors from 1007 patients (at least 1 gene tested) and 733 patients (tested for 10 genes) from 2009 - 2012. Results revealed 466 of 733 patients (64\%) tested positive for an oncogenic driver. ${ }^{13}$ Targeted therapy was initiated in $28 \%$ of the patients (275 of 1007 patients). Mean survival for those receiving genotype-directed targeted therapy was 3-5 years compared to 2.4 years for those patients that did not receive the targeted therapy. ${ }^{13}$ The authors concluded that "although individuals with drivers receiving a matched targeted agent lived longer, randomized trials are required to determine if targeted therapy based on oncogenic drivers improves survival."13 In addition, the authors predict that as more targeted therapies are investigated in clinical trials in patients with lung carcinoma, the number of individuals treated based on their genotype will increase. ${ }^{13,14}$

\section{Human Interest Story}

An interesting story highlighted in 2012 in the New York Times entitled, "In Treatment for Leukemia, Glimpses of the Future" ${ }^{15}$ a genetic researcher working on acute lymphoblastic leukemia (ALL) was diagnosed with the same blood cancer he spent his life studying. There was no known treatment and his condition was getting worse over time. His research team decided to sequence the genome in his cancer cells and compare the results to the genes of his normal cells. ${ }^{15}$ In addition, they analyzed his RNA in an attempt to determine what was going on at the genetic level. What they found was the cancer DNA had multiple mutations which nothing could be done about it however, his RNA revealed a normal gene, fetal liver tyrosine kinase 3 (FLT3), was extremely active in the leukemic cells. ${ }^{15}$ This gene, under normal circumstances, allows cells to multiply and grow however, in an overactive state, it could cause the rapid proliferation of the leukemic cells. Fortunately a drug was available called Sunitinib or Sutent which is a FLT3 inhibitor. ${ }^{15}$ Although approved for kidney cancer treatment, the patient started the drug and within days all his blood counts returned to normal. Multiple tests were done to confirm the results such as flow cytometry and FISH, but all revealed the same result; the cancer cells were no longer evident. ${ }^{15}$ At the time the article was published in 2012, he was in remission. This case is just one of many examples in the literature where treatment was targeted based on the genetic make-up of the cancer or the treatment regimen was designed after identifying multiple mutations on the individual's own DNA or in this case RNA.

For an overview regarding the use of NGS in the clinical molecular diagnosis of cancer refer to the article published in Medical Laboratory Observer (MLO) by Rhea et al. which can be found at the following link: http://www.mlo-online.com/articles/201112/nextgeneration-sequencing-in-the-clinical-moleculardiagnosis-of-cancer.php. ${ }^{16}$

\section{New Cancer Panel for Personalized Medicine}

The introduction of a new cancer panel was recently announced by the Mayo Clinic in April 2014. This 50 gene cancer panel test will aid in personalizing cancer treatment based on the genetic make-up of one's own tumor. ${ }^{17}$ The test, abbreviated CANCP, stands for Solid Tumor Targeted Cancer Gene Panel by NextGeneration Sequencing and can "scan specific regions in 50 genes known to affect tumor growth and response to chemotherapy." ${ }^{17}$ According to Dr. Axel Grothey, "every patient's cancer is different, and oncology is moving away from treating cancer based on its location 
in the body in favor of selecting the best medication for the individual patient based on molecular changes in the tumor." ${ }^{17}$ This test is an exciting new development which is available to Mayo Clinic patients and to laboratories worldwide through Mayo Medical Laboratories. ${ }^{17}$

\section{Next Generation Sequencing in Personalized Medicine and Ethics}

Prior to the completion of the human genome project, clinicians treated a patient's illness based on the medication available for that disease. In short, clinicians treat the disease and not the patient since it is unknown whether the patient will respond or have an overall positive effect from the therapy administered. With the advent of DNA sequencing techniques and the completion of the human genome project, clinicians now have the ability to treat and monitor a patient based on their own genetic make-up. Since each patient has a unique genome, clinicians can no longer have the mindset that "one size fits all" in regards to therapy. The promise of personalized medicine in diagnosing, treating, and monitoring an individual will not only be beneficial to the patient's prognosis and overall quality of life but has the potential to considerably lower healthcare costs and reduce hospital stays. ${ }^{18}$

The efficacy of medications prescribed is approximately $50 \%$ to $60 \%$ for most disorders and is only $20 \%$ for cancer regimens. ${ }^{18}$ The Agency for Health Care Research and Quality (AHRQ) reported that "unsafe and ineffective drugs cause avoidable deaths; adverse reactions, many of which result in costly hospitalizations; and wastage resulting from discarding medications that don't work and, that adverse reactions result in more than 770,000 injuries and deaths each year and cost up to $\$ 5.6$ million per hospital, depending on size." ${ }^{19}$

Once genetic testing became available, individuals were concerned that their insurance carrier would no longer cover them and/or they would be terminated from their workplace if a genetic disorder were discovered. This concern was resolved by the passing of the Genetic Information Nondiscrimination Act of 2008 (GINA). This act "protects Americans from discrimination based on their genetic information in both health insurance (Title I) and employment (Title II). Title I amends the Employee Retirement Income Security Act of 1974
(ERISA), the Public Health Service Act (PHSA), and the Internal Revenue Code (IRC), through the Health Insurance Portability and Accountability Act of 1996 (HIPAA), as well as the Social Security Act, to prohibit health insurers from engaging in genetic discrimination." ${ }^{20}$ Additional information regarding this act can be found at the following link: https://www.genome.gov/10002077.

Although GINA is in place to protect the patient, concern still exists over whether insurance companies will eventually require genetic testing be performed on a patient who is not responding to a particular drug or treatment. This testing would attempt to avoid additional costs of "trial and error prescribing." 18 If genetic testing does become a requirement for insurance payment, the patient no longer has the choice to undergo genetic testing and may be refused empirical treatment since it may have no effect or a negative effect against their condition. Since personalized medicine does require that a patient's genome or portions of it be sequenced it may leave an individual vulnerable to learning information about themselves they may not want to know. Whether this happens or not, there is always the possibility that insurance companies will want this information not to discriminate against but to pay for the appropriate treatment. ${ }^{18}$

Another issue when obtaining genetic information lies in the hands of the individuals testing and reviewing the results. Researchers are not required to report genetic mutations found while looking for a specific mutation. Ethically, if a researcher or a clinical laboratory scientist stumbles upon a genetic variant that can have lifethreatening consequences for the subject or patient, should this finding not be communicated to the patient? Is it not the individual's right to know this information? Maybe the individual does not want to know this information. This certainly presents an ethical dilemma. Recently several committee members from four groups/consortiums published "Suggested Guidelines for Returning Sequencing Results to Research Participants" in the American Journal of Human Genetics (in press May 2014). ${ }^{21}$ These recommendations attempt to provide guidance on what a researcher should do if they discover a genetic result that could have a detrimental effect on the participants health. Another issue is whether this information should be reported to the parents if a child cannot consent to 
receiving this information. The committee concluded the following, "during the consent process, parents should be offered the choice of having adult-onset actionable incidental findings returned, along with counseling. However, in cases where one or both parents are already known to carry variants related to adult-onset conditions that do not change clinical management in childhood, the discovery of the same variant in the child does not need to be returned since the family already is aware of its presence." ${ }^{21}$ This statement opens the door to much controversy and as well as moral obligations. ${ }^{21}$ Committee members also recommend the results be confirmed by a CLIA compliant laboratory before results are returned. Committee members believe this recommendation will generate additional controversy. ${ }^{21}$ Regardless of the controversy, guidelines should be published to standardize the procedure when genetic results are obtained that could have an overall ill effect on the patient.

\section{Summary}

An entire series could be dedicated to the topic of ethics in personalized medicine. Due to the advancements in NGS and genetic testing, personalized medicine is no longer something that will occur in the future, the reality is upon us now. Sequencing an individual's genome can have a substantial impact on the patient's treatment and overall quality of life. However, this can open "Pandora's box" especially if an individual does not want to know the information obtained. In addition, will insurance companies require genetic testing in order to pay for a targeted treatment? If the patient refuses to have the genetic testing, will they have to pay for their treatment out of pocket? In the human interest story presented, the researcher and his team discovered over activity of the FTL3 protein through RNA sequencing which resulted in rapid proliferation of his leukemic cells. He identified a drug marketed for advanced kidney cancer which was a FTL3 inhibitor. However, his insurance company refused to pay for the drug because it was not a known treatment for his condition of ALL. He incurred numerous out of pocket expenses in order to go into remission. Was it unethical for the insurance company to not pay for a treatment that ultimately worked but was not marketed or FDA cleared for his type of leukemia? There are so many questions and concerns when personalized medicine is implemented. Only time will tell the effects next generation sequencing and its role in personalized medicine will have in the future.

\section{ACKNOWLEDGEMENT}

The author would like to acknowledge Robert Donnelly, $\mathrm{PhD}$ for his guidance and support in the development of this article.

NOTE: The author is not endorsing any particular company or product and has no financial gain or otherwise interest in the products presented.

\section{REFERENCES}

1. Personalized Medicine. Available from http://ghr.nlm.nih.gov/ glossary=personalizedmedicine. Accessed 2014 May 21.

2. Ion AmpliSeq ${ }^{\mathrm{TM}}$ Ready-to-Use Panels. Available from http://www.lifetechnologies.com/us/en/home/life-science/se quencing/dna-sequencing/targeted-sequencing/targeted-se quencing-ion-torrent-next-generation-sequencing.html. Accessed 2014 March 31.

3. Ion AmpliSeq ${ }^{\mathrm{rm}}$ Custom DNA Panels. Available from http://www.lifetechnologies.com/us/en/home/life-science/se quencing/next-generation-sequencing/ion-torrent-next-genera tion-sequencing-workflow/ion-torrent-next-generation-sequenc ing-select-targets/ampliseq-target-selection/custom-panels.html. Accessed 2014 March 31.

4. Carlson B. Next Generation Sequencing: the next iteration of personalized medicine. Biotechnology Healthcare. 2012;9(2):21-5.

5. Personalized Medicine. Available from http://www.cancer.gov/ dictionary?CdrID=561717. Accessed 2014 May 20.

6. Whole Genome Sequencing. Available from http://www. lifetechnologies.com/us/en/home/life-science/sequencing/dnasequencing/whole-genome-sequencing.html. Accessed 2014 May 20.

7. Guan YF, Li GR, Want RJ, Yi YT, Yang L, and Jian D. Application of next-generation sequencing in clinical oncology to advance personalized treatment of cancer. Chin J Cancer. 2012;31(10):463-70.

8. Dewey FE, Grove ME, Pan C, Goldstein BA, Bernstein JA, Chaib $\mathrm{H}$, et al. Clinical interpretation and implications of whole-genome sequencing. JAMA. 2014;311(10):1035-45.

9. Challenges remain before docs use whole-genome sequencing. Available from http://www.reuters.com/article/ 2014/03/11/uschallenges-genome-idUSBREA2A1SV20140311. Accessed 2014 May 20.

10. Welch JS, Westervelt P, Ding L, Larson DE, Klco JM, Kulkarni $S$, et al. Use of whole genome sequencing to diagnose a cryptic fusion oncogene. JAMA. 2011;305(15):1577-84.

11. Hill BT, Copelan EA. Acute myeloid leukemia: when to transplant in first complete remission. Curr Hematol Malig Rep. 2010;5(2):101-8.

12. ATRA. Available from http://chemocare.com/chemotherapy/ drug-info/atra.aspx\#.U30CRiiooTA. Accessed 2014 May 20.

13. Kris MG, Johnson BE, Berry LD, Kwiatkowski DJ, Iafrate AJ, Wistuba II, et al. Using multiplexed assays of oncogenic drivers in lung cancers to select targeted drugs. JAMA. 
2014;311(19):1998-2006.

14. Study shows improved survival for lung cancer patients receiving treatment based on genotype. Available from http://www.ge nomeweb.com/print/1391311. Accessed 2014 May 21.

15. In treatment for leukemia, glimpses of the future. Available from http://www.nytimes.com/2012/07/08/health/in-gene-se quencing-treatment-for-leukemia-glimpses-of-the-future.html? pagewanted=1\&_r=1. Accessed 2014 May 21.

16. Reha JM, Singh HV, Molinaro RJ. Next generation sequencing in the clinical molecular diagnosis of cancer. Available from http://www.mlo-online.com/articles/201112/next-generationsequencing-in-the-clinical-molecular-diagnosis-of-cancer.php. Accessed 2014 May 21.

17. Mayo Clinic launches 50-gene cancer panel test. Available from http://newsnetwork.mayoclinic.org/discussion/mayo-clinic- launches-50-gene-cancer-panel-test. Accessed 2014 May 21.

18. Personalized medicine part 2: ethical, legal, and regulatory issues. Available from http://www.ncbi.nlm.nih.gov/pmc/ articles/PMC2993070/. Accessed 2014 May 22.

19. Agency for Health Care Research and Quality (AHRQ) Research in Action. Reducing and preventing adverse drug events to decrease hospital costs. Available from http://www. ahrq.gov/research/findings/factsheets/errorssafety/aderia/index.html. Accessed 2014 May 22.

20. Genetic Discrimination. Available from https://www.genome. gov/10002077\#al-2. Accessed 2014 May 21.

21. Committee members suggest guidelines for returning sequencing results to research participants. Available from http://www.genomeweb.com/sequencing/committee-memberssuggest-guidelines-returning-sequencing-results-research-parti. Accessed 2014 May 22.

\section{ASCLS CERTIFICATION MAINTENANCE}

\section{EASILY EARN CE CREDIT!}

\section{CERTIFICATION MAINTENANCE MEMBERSHIP (CMM)}

Renewing or joining members can select the CMM option which provides a subscription for 12 hours of online P.A.C.E. ${ }^{\circledR}$ approved continuing education (CE) for only $\$ 55$ plus national and state dues.

CMM is a 1-year subscription with ASCLS' partner, MediaLab, Inc.

The 12 hours cover designated discipline areas for the Board of Certification (BOC) Certification Maintenance Program.

\section{CERTIFICATION MAINTENANCE MEMBERSHIP}

Upgrade to unlimited hours of CE and select the course you want for $\$ 95$ plus national and state dues.

CMMP is a 1-year subscription with ASCLS' partner, MediaLab, Inc.

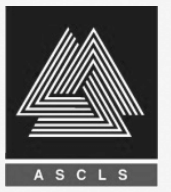

Go to www.ascls.org/cmm for more information. 